\title{
Sometimes (What Seems to Be) A Heart Attack Is (Really) A Pain In The Neck
}

\author{
Anthony A. Mork, MD, Scott M. W. Haufe, MD, and William B. Yancey, MD,
}

A 31-year-old patient complained of severe crushing chest pain that radiated to his left arm and jaw. After admission to the hospital, tests revealed a normal electrocardiogram, normal treadmill, normal coronary arteriogram, and normal cardiac enzymes. However, the patient continued to have pain, which was relieved by sublingual and intravenous nitroglycerine. He was discharged from the hospital with a diagnosis of "musculoskeletal" chest pain, taking nonsteroidal anti-inflammatory drugs, muscle relaxants, and narcotics. Two weeks later, the patient returned with worsening symptoms. Cardiac work-up was again negative. Thoracic and cervical spine radiographs were ordered for possible discogenic pain. After abnormalities were found on cervical radiographs, magnetic resonance imaging (MRI) was ordered, and the patient was referred to an orthopedic surgeon. Further work-up revealed a herniated disk at $\mathrm{C} 6-\mathrm{C} 7$, with radicular pain. Surgery on the suspect disk totally relieved the patient's pain. (J Am Board Fam Pract 2004;17:74-7.)

Chest pain is inherently difficult to diagnose, even when it follows a typical pattern. Many causes of chest pain can be lethal within minutes to hours. They include myocardial infarction, pulmonary embolus, pneumothorax, ruptured esophagus, and bleeding peptic ulcer, to name a few.

Making a final diagnosis, without loss of blood, obvious electrocardiogram (EKG) changes, or obvious changes in arterial oxygen saturation, cardiac enzymes, or blood pressure is sometimes challenging. Many patients are admitted with suspected myocardial infarction or acute peptic ulcer but are proven to have other diagnoses.

One source of chest pain rarely responsible for simulating a myocardial infarction is the cervical spine. ${ }^{1,2}$ Segmental nerves originating from the C3-4 interspace to the C7-T1 interspace innervate dermatomes from the jaw to the mid-chest, just above the nipples anteriorly and mid-scapulae posteriorly. The space on the thorax between the C5 and $\mathrm{T} 1$ dermatomes anteriorly was the location of segmental dermatomes for C6 to C8 in our segmented ancestors. It makes sense that there may be some vestigial remnants of those nerve endings in humans. Pain patterns associated with the nerves

Submitted, revised, 3 October 2003.

From Microspine, Inc., Defuniak Springs, Florida. Address correspondence to William B. Yancey, MD, 19 Buddy Street, Santa Rosa Beach, FL 32459 (e-mail: wbyancey@ earthlink.net). originating in the cervical spine seem to closely match the dermatomes.

In addition, these segmental nerves innervate muscle, fascia, bone, and organs in areas of the body associated with their dermatomes. Each nerve is not totally independent; they communicate with one another. There is also some natural variation in anatomy, making it sometimes difficult to decide which nerve is responsible for a particular area of the body. One representation of the C6, C7, and C8 dermatomes is shown in Figure 1. Radicular pain from any of these segmental nerves can be mistaken for cardiac pain.

\section{Case Report}

\section{History}

Late one afternoon, a 31-year-old grocery delivery driver presented himself to the emergency department (ER) complaining of left-sided chest pain. The ER encounter form notes that patient had pain radiating to his left jaw and down his left arm. The pain had been present since early that morning. The patient also complained of a cough and the production of bloody sputum. The triage nurse's notes state the patient's pain increased with deep respiration or strenuous activity. She also noted the patient was sweaty and dizzy. The physician's history indicated that the results of a previous glucose tolerance test taken by the patient were interpreted as hypoglycemia, that the patient was a 2 pack-perday smoker. The patient did a lot of heavy lifting- 


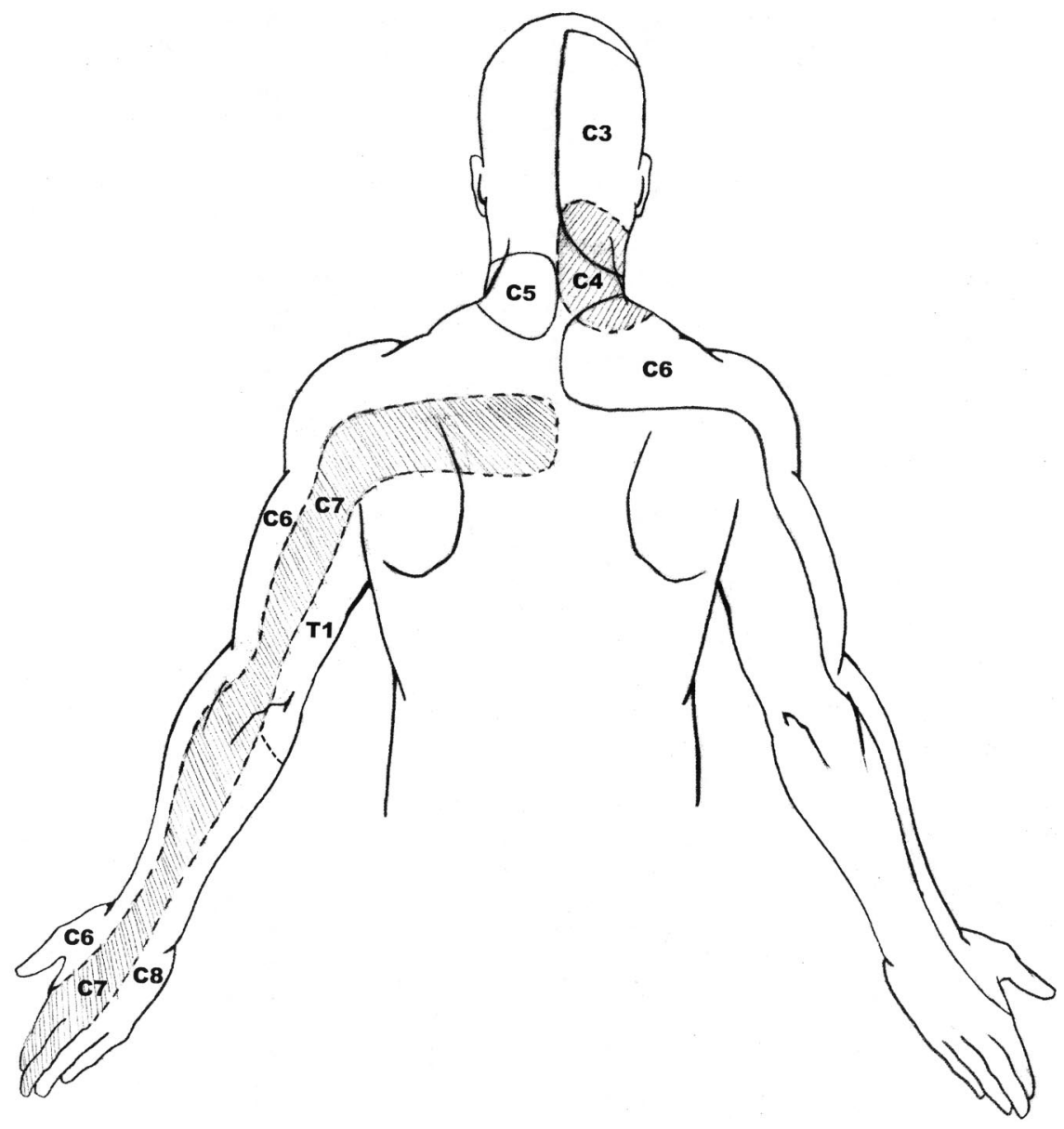

Figure 1. C2 to $\mathrm{C8}$ pain patterns as found in actual practice, secondary to cervical nerve root compression and/or facet injury.

upwards of 100 pounds at a time. The patient first noted pain when getting into his truck after a delivery. There were no references in the chart concerning radicular pain, numbness, or extremity weakness. Physical examination revealed normal throat, irritated left nasal mucosa, clear lungs, and soft abdomen. No neurological examination was recorded. Chest radiograph, EKG, creatine kinase, electrolytes, prothrombin/partial thromboplastin times, and complete blood count were apparently within normal limits. Determination of serum cardiac troponins was apparently thought to be unnecessary. The patient was treated with clarithromycin (Biaxin) for bronchitis and cyclobenzaprine (Flexeril) for pain. The emergency physician discharged him home.

Follow-up with his family physician and his physician's assistant led to a treadmill test. This test was completed 8 days later. The patient reached level 4 on the Bruce protocol. At that point, the patient's pain recurred. The pain was relieved with sublingual nitroglycerin (NTG).

The following day, the patient returned to the ER complaining of severe chest pain relieved with nitroglycerin spray administered by emergency medical technicians. He was admitted to the hospital intensive care unit (ICU) on oxygen, heparin, and NTG. Routine lab work, including CBC, electrolytes, troponins, and creatine kinase were negative. Family history included diabetes and hypertension. The patient continued to smoke. There was no change in chest radiograph or EKG. Based solely on the patient's continued complaints of chest pain relieved by NTG, the patient was transferred to a larger hospital.

Four days after readmission, the patient underwent cardiac catheterization. History taken at that time confirmed the patient had no previous cardiac complaints. Also noted was a gripping, left parasternal chest pain, worse on exertion, that ra- 
diated to arms and shoulder, in addition to the abnormal stress test. Cardiac catheterization demonstrated an ejection fraction of $62 \%$, with essentially normal coronary arteries. The patient was discharged with a diagnosis of musculoskeletal pain, treated with ketorolac (Toradol), rofecoxib (Vioxx), and hydrocodone/acetaminophen (Lortab). He was thought to be anxious and was counseled to quit smoking.

Approximately 1 month after initial presentation, the patient returned to the ER with a recurrence of his pain. It radiated to his left arm and was worse on inspiration. He was admitted to the ICU. Lab studies, EKG, etc, were normal. Thoracic and cervical spine radiographs were ordered. The patient left the hospital against medical advice after being told that he could not smoke.

The patient again saw his family physician and physician's assistant for follow-up. The thoracic radiographs taken in the hospital were interpreted as normal. Cervical $\mathrm{x}$-rays were read as straightening of the cervical spine consistent with spasm, and minimal degenerative changes noted, from $\mathrm{C} 3$ to C6. These abnormalities led his physician to order MRI of the cervical and thoracic spine. Interpretation of the MRI read: ". . . mild foraminal stenosis bilaterally at C6-7. . C3-4. Midline disk bulge at both levels ... spinal canal stenosis at C6-7. Early disk degeneration ... T8-9 . . T9-10 . . T12L1." The patient's family physician then referred the patient to an orthopedic surgeon.

The senior author, an orthopedic surgeon, examined the patient 3 weeks later. History noted at that time included sudden onset of shoulder and arm pain after a delivery, on the day of initial presentation to the ER, with numbness and pain along the ulnar aspect of the left arm, and left 4th and 5th fingers (present since before initial ER visit). In addition, the patient complained of numbness and pain on the left side of his face, the base of his neck, and the left side of his neck. The pain was made worse by moving his head. It radiated to his xiphoid proces,s where it felt like a spike being driven into his chest. By this time, the patient had been placed on meperidine (Demerol) and oxycodone (OxyContin), in addition to his other medications.

\section{Physical Examination}

The patient was right-handed. Range of motion of the cervical spine was slightly decreased in flexion and exhibited $20^{\circ}$ of extension, $20^{\circ}$ lateral flexion bilaterally, and $75^{\circ}$ of rotation bilaterally. Deep tendon reflexes (DTRs): triceps, absent bilaterally; biceps, absent on left, $1+$ on right; brachioradialis, absent on left, trace on right. Decreased pinprick sensation on left fifth finger and ulnar side of the fourth finger.

\section{Imaging}

(Interpreted by the senior author) cervical spine radiograph: foraminal stenosis at $\mathrm{C} 2-3$; no acute changes. Cervical spine MRI: disk bulges at $\mathrm{C} 3-4$ and C6-7. Foraminal stenosis at $\mathrm{C} 3-4$ on left, and C6-7 on the left. Mild central stenosis at C3-4 and mild central stenosis at C6- 7 .

\section{Treatment}

Shortly thereafter, the second author, an anesthesiologist, performed a cervical epidural injection on the patient at the C6-7 level on the left. Two milliliters of lidocaine (Xylocaine) and $80 \mathrm{mg}$ of triamcinolone (Aristocort) were injected. The patient had 3 days of total pain relief after the injection. His pain returned gradually to its former intensity, in the same locations.

Four months after the initial ER visit, the senior author performed an arthroscopic, micro-discectomy at the C6-7 level, on the left. This procedure is relatively new. It is done through an anterior approach to decompress the disk without a fusion. Using fiberoptic visualization, the deteriorated disk fragments were removed, decompressing the spinal cord and segmental nerve within the left foraminal canal.

\section{Follow-Up}

On the first day after surgery, the patient had no chest pain or left arm pain or numbness. He did have surgical wound discomfort.

One month after surgery, the patient insisted on being returned to normal duty, without restrictions, and against medical advice. He stated he was absolutely asymptomatic.

Six months after surgery, the patient demonstrated increased range of motion of his neck, normal strength in both upper extremities, and the return of normal reflexes.

At 1 year, the patient complained of occasional pain in his left posterior arm and anterior chest, associated with strenuous activity. He also has some posterior neck pain, which may be spinous process 
pain and/or discogenic, in that he still has the bulging disk at $\mathrm{C} 3-4$.

\section{Discussion}

There are multiple sources for anterior chest pain. After the most lethal possibilities are ruled out, it makes sense to consider the cervical and thoracic spine as a source. Taking a good history, including a brief neurological history, and performing a good physical examination, including a neurological examination, may give clues to musculoskeletal sources of chest pain. In a young man with no prior cardiac history, negative EKG changes, and negative cardiac enzymes, such an approach might have saved this patient an admission, a treadmill test, and a cardiac catheterization.

We thank Kyle Cunningham for the illustration.

\section{References}

1. Rothman R, Marvel J. The acute cervical disk. Clin Orthop Rel Res 1975;109:59-68.

2. Dwyer A, April C, Bogduk N. Cervical zygapophyseal joint pain patterns. I: a study in normal volunteers. Spine 1990;15:453-7. 